\title{
Immune checkpoint inhibitor induced autoimmune encephalitis in a patient with metastatic melanoma
}

\author{
Nivedita Sudhekar, Binoy Yohannan, Mark Feldman* \\ Department of Internal Medicine, Texas Health Presbyterian Hospital, Dallas, Texas, United States
}

Received: March 2, 2020

Accepted: June 25, 2020

Online Published: July 14, 2020

DOI: $10.5430 /$ crim.v7n3p1

URL: https://doi.org/10.5430/crim.v7n3p1

\begin{abstract}
Background: Immune checkpoint inhibitors have changed the therapeutic milieu for patients with metastatic melanoma. However, their use may promote autoimmunity in virtually any organ in the body due to the blockade of intrinsic immune down regulators such as cytotoxic T-lymphocyte antigen- 4 (CTLA-4), programmed cell death 1 (PD1) or its ligand (PDL1). Immune mediated adverse neurological events are rare with these agents, however, and are seen in $<1 \%$ of treated patients. We report a patient with immune checkpoint inhibitor associated autoimmune encephalitis, with complete clinical recovery after treatment. Case Report: A 49-year-old female with metastatic melanoma currently on nivolumab therapy but recently on ipilimumab/nivolumab combined therapy presented with a new headache. She also reported associated confusion, loss of balance, personality changes and language difficulty. Magnetic resonance imaging of the brain did not reveal any evidence of metastasis, infarct, meningitis, or encephalitis. Lumbar puncture revealed an elevated protein level and pleocytosis, with a normal glucose level. She was started on empiric glucocorticoid therapy with a presumptive diagnosis of immune checkpoint inhibitor associated autoimmune encephalitis. She improved considerably by day 3 of treatment with complete resolution of neurological symptoms by day 5 .

Conclusion: Immune checkpoint inhibitors are increasingly important in cancer immunotherapy as they can cause sustained remissions in patients with metastatic melanoma and other malignancies. Because these drugs block immune-regulatory targets, they can lead to enhanced activation of immune system resulting in immune-related adverse events. Autoimmune encephalitis is a rare immune-related adverse event associated with immune checkpoint inhibitors. The incidence of autoimmune encephalitis is higher with combination or sequential CTLA-4 (ipilimumab) and PD1(nivolumab) inhibitor therapy than with monotherapy. With more widespread use of immunotherapy, it is important for clinicians to be aware of this rare and reversible cause of encephalitis. Early recognition and prompt initiation of immunosuppressive therapy with glucocorticoids is essential to enhance neurological recovery.
\end{abstract}

Key Words: Encephalitis, Immune related adverse effects, Immune checkpoint inhibitor, Nivolumab, Ipilimumab

\section{BACKGROUND}

Immune checkpoints are important endogenous inhibitory pathways that are crucial for maintaining self-tolerance and modulating immune responses. ${ }^{[1]}$ Immune checkpoint inhibitors (ICIs) have emerged as one of the most efficacious therapeutic regimen that enhance anti-tumor $\mathrm{T}$ cells, improv- ing clinical outcomes and survival in patients with metastatic melanoma and several other metastatic malignancies including non-small cell lung cancers and renal cell cancers. ${ }^{[2]} \mathrm{Al}-$ though these novel immunotherapy drugs have changed the treatment paradigm for these tumors, they can cause immunerelated adverse events due to dysregulated $\mathrm{T}$ cells targeting

\footnotetext{
*Correspondence: Mark Feldman; Email: MarkFeldman@texashealth.org; Address: Department of Internal Medicine, Texas Health Presbyterian Hospital, Dallas, Texas 75231, United States.
} 
multiple organs such as the liver, skin, endocrine system, gut and even the nervous system. ${ }^{[3]}$ Immune-related neurological adverse events with ICIs are rare $(<1 \%) .{ }^{[4]}$ Here, we present a case of autoimmune encephalitis following treatment with ipilimumab and nivolumab.

\section{CASE REPORT}

A 49-year old Caucasian female with metastatic melanoma, currently on nivolumab monotherapy, presented with a new headache, confusion, incomprehensible speech with word finding difficulty and loss of balance. Her family also noticed socially inappropriate behaviors such as urinating on the carpet. She and her family denied fever, chills, vomiting, neck stiffness, photophobia, head trauma or seizures. Five months earlier, she had been diagnosed with unresectable metastatic melanoma presenting as cervical and thoracic lymph node metastasis with unknown primary. The tumor was not associated with BRAF, KIT, NRAS mutations. Treatment initiated with nivolumab (PD1 inhibitor) and ipilimumab (CTLA-4 inhibitor) for three cycles. Subsequently, she developed immune related grade 3 hepatotoxicity (AST 593, ALT 398, Alkaline phosphatase 926 and total bilirubin of 5.9), that was treated successfully with glucocorticoids after discontinuation of both of the ICIs. One month prior to admission, she was restarted on nivolumab monotherapy. Other medical problems included anxiety, depression, migraine, hypothyroidism (acquired) prior to melanoma diagnosis, and gastroesophageal reflux disease. She had no family history of cancer. She is a former smoker and used alcohol and marijuana occasionally for her migraine. In addition to her treatment with nivolumab $240 \mathrm{mg}$ every 2 weeks, she was also on buspirone $30 \mathrm{mg}$ daily, duloxetine $30 \mathrm{mg}$ daily, quetiapine $50 \mathrm{mg}$ at bedtime, propranolol $20 \mathrm{mg}$ twice daily, gabapentin $600 \mathrm{mg}$ three times daily, omeprazole $20 \mathrm{mg}$ daily, np thyroid $60 \mathrm{mg}$ tab daily and omeprazole $20 \mathrm{mg}$ daily. No recent changes in these medications. Her other concurrent medical conditions were stable.

Vital signs were within normal limits. Her general physical examination was unremarkable. She was co-operative and alert but oriented to self only. Ophthalmological examination revealed $3 \mathrm{~mm}$ reactive pupils with full conjugate extraocular movements and no nystagmus or ptosis. Fundoscopic examination revealed normal disk margins and retinal vascularity. She had difficulty with comprehension, recollection, following commands, and performing serial seven subtractions. She could not spell "world" backwards. She replied with only one or two words to any question asked. She had intermittent misuse of words and significant word finding difficulty. Her short-term memory was significantly impaired. Cranial nerves 2 through 12 were intact. Motor ex- amination showed 5/5 muscle power in both upper and lower extremities in the proximal and distal groups of muscles. Her reflexes were symmetric and normal. Her sensory system examination was normal. Cerebellar examination revealed ataxia and dysmetria. She did not have any meningeal signs.

Her complete blood count, coagulation profile and comprehensive metabolic panel were normal. Urine drug screen (toxicology) was positive only for cannabinoids. Serum vitamin B12 level was 779 (213 - $816 \mathrm{pg} / \mathrm{ml})$. Her TSH, free T4 and free T3 levels were all decreased with consideration mainly for acquired transient central hypothyroidism secondary to non-thyroidal illness but difficult to differentiate from central hypothyroidism secondary to treatment with ICIs. Levels were $<0.1(0.5-5 \mathrm{uIU} / \mathrm{ml}), 0.64(0.70-1.48 \mathrm{ng} / \mathrm{dl})$ and 1.69 $(1.71-3.71 \mathrm{pg} / \mathrm{ml})$, respectively. Computerized tomography (CT) of the head and magnetic resonance imaging (MRI) of the brain with and without contrast were normal. In consultation with neurology, EEG was deferred. Her lumbar puncture revealed a normal opening pressure, clear fluid and cerebrospinal fluid (CSF) analysis as described in Table 1. An autoimmune encephalitis panel was negative as described in Table 2.

She was started on empiric intravenous methylprednisolone (1 gm per day) with a presumptive diagnosis of ICI-induced autoimmune encephalitis. Antimicrobials and anti-epileptics were deferred. Nivolumab was discontinued. On day 2 of glucocorticoid therapy, her cognition and speech gradually improved and she was making meaningful sentences. By day 4 , she was alert, oriented and back to her baseline mental status and function. Her memory and cognitive deficits completely resolved. She received 5 daily doses of intravenous methylprednisolone and was discharged home with no residual neurological deficits on prednisone $80 \mathrm{mg}$ per day with plans for prolonged steroid taper.

Table 1. Cerebrospinal fluid (CSF) analysis: CSF VDRL, HSV PCR, cryptococcal antigen, cytology and cultures were negative.

\begin{tabular}{ll}
\hline Test (reference range) & Value in this patient \\
\hline Protein $(15-40 \mathrm{mg} / \mathrm{dl})$ & $155 \mathrm{mg} / \mathrm{dl}$ \\
$\operatorname{IgG}(0.48-5.86 \mathrm{mg} / \mathrm{dl})$ & $9.88 \mathrm{mg} / \mathrm{dl}$ \\
IgG index ratio $(0.28-0.66)$ & 0.46 \\
Glucose $(40-70 \mathrm{mg} / \mathrm{dl})$ & $49 \mathrm{mg} / \mathrm{dl}$ \\
WBC count $\left(0-5 / \mathrm{mm}^{3}\right)$ & $77 / \mathrm{mm}^{3}$ \\
& $(43 \%$ Lymphocytes, \\
& $56 \%$ Neutrophils $)$ \\
Oligoclonal bands (negative) & negative \\
Myelin basic protein $(0-5.50 \mathrm{ng} / \mathrm{ml})$ & $4.43 \mathrm{ng} / \mathrm{ml}$ \\
\hline
\end{tabular}


Table 2. Autoimmune encephalitis panel on cerebrospinal fluid (CSF): contactin-associated protein-like 2, anti Ma-2, anti-Hu, R1 receptor Ab were not tested.

\begin{tabular}{lll}
\hline Test & Result & Reference value \\
\hline NMDA-R Ab CBA & Negative & Negative \\
VGKC-complex Ab IPA & 0 nmol/L & $0-0.02 \mathrm{nmol} / \mathrm{L}$ \\
LGI1-IgG CBA & Negative & Negative \\
CASPR2-IgG CBA & Negative & Negative \\
GAD65 Ab Assay & $0.01 \mathrm{nmol} / \mathrm{L}$ & $\leq 0.02 \mathrm{nmol} / \mathrm{L}$ \\
GABA-B-R Ab CBA & Negative & Negative \\
AMPA-R Ab CBA & Negative & Negative \\
ANNA-1,2,3 & $<1: 2$ & Titre $<1: 2$ \\
PCA -1,2,3 & $<1: 2$ & Titre $<1: 2$ \\
Amphiphysin Ab & $<1: 2$ & Titre $<1: 2$ \\
CRMP-5-IgG & $<1: 2$ & Titre $<1: 2$ \\
\hline
\end{tabular}

Note. CBA-cell based assay, Ab- antibody, R- receptor, IPA- immunoprecipitation assay.

\section{Discussion}

Although ICIs have improved the treatment of metastatic melanoma and other cancers, they may give rise to a unique set of immune related adverse effects (irAE) due to uninhibited function of $\mathrm{T}$ cells targeting various organs. Immune related neurotoxicity is rare, affecting $<1 \%$ of ICI treated patients, and can involve both the central and peripheral nervous system. ${ }^{[5,6]}$ Immune related neurotoxicity is observed more frequently in patients treated with a combination of ipilimumab and nivolumab with a reported incidence of $14 \% .{ }^{[6]}$ An Analysis of 59 trials totaling 9,208 patients reported the overall incidence of neurologic irAEs to be $3.8 \%$ for CTLA4 inhibitors, $6.1 \%$ for single agent PD1 inhibitors and $12 \%$ for combination of both. ${ }^{[7]}$ The most common immune mediated neurological adverse events are aseptic meningitis, paresthesia/ peripheral neuropathy, parkinsonism, steroidresponsive confusion, myasthenia gravis and Guillian-Barre syndrome. ${ }^{[8,9]}$ Autoimmune encephalitis is quite rare, is seen more commonly with a combination of ipilimumab and nivolumab, and is mediated by cytotoxic $\mathrm{T}$ cells targeting neuronal antigens. ${ }^{[6,8]}$ The immune mediated neurotoxicity is commonly seen with in the first 4 months after initiation of therapy but can be seen at any stage in the treatment course. ${ }^{[6]}$ Our patient was started on nivolumab (PD1 inhibitor) and ipilimumab (CTLA-4 inhibitor) combination therapy for three cycles at diagnosis. Due to her development of grade 3 hepatotoxicity, she was off treatment for one month and was subsequently started on nivolumab monotherapy. She presented to our hospital with encephalitis after one month of nivolumab. Therefore, her disease could possibly be attributed to the prior ipilimumab and nivolumab combination therapy even though she was on nivolumab monotherapy at the time of her presentation. Patients with the less common autoimmune encephalitis may present with nonspecific signs

Published by Sciedu Press and symptoms characterized by headache, fever, confusion, lethargy, disorientation, memory impairment, drowsiness, hallucinations, seizures and poor concentration. ${ }^{[10,11]}$

The exact mechanism of immune related neurotoxicity is not clear. T-cells, autoantibodies and / or cytokines may play a role. Inflammation around endoneural blood vessels and perineural edema also may contribute. ${ }^{[12]}$ PD-1(the target of nivolumab) is also expressed on B cells and PD-1 blockade has been shown to enhance $\mathrm{B}$ cell activation and proliferation, thereby predisposing to autoantibody-mediated disease. ${ }^{[13]}$ Immune-mediated encephalitis is usually a diagnosis of exclusion and can occur at any time during therapy. In patients with new-onset neurologic signs or symptoms, alternate diagnoses such as metastatic disease, CNS infection, paraneoplastic encephalitis and toxic/metabolic syndromes should be considered and carefully excluded. A lumbar puncture and CSF analysis is probably the most helpful tool to support the diagnosis. An elevated CSF protein level with either neutrophilic or lymphocytic pleocytosis supports an immune mediated inflammatory disease process. ${ }^{[7,8]}$ In most cases, no specific onco-neuronal autoantibodies are isolated, but rarely paraneoplastic antibodies to contactin-associated protein-like 2, Ma-2, Hu and the NMDA receptor have been implicated. In our patient, the NMDA receptor antibody test was negative, but antibodies to contactin-associated protein-like 2 , $\mathrm{Ma}-2, \mathrm{Hu}$ were not included in the general autoimmune encephalitis panel that was performed. NMDA receptors are expressed on the surface of melanocytes and auto-antibodies against these NMDA receptors in melanoma may cross-react with NMDA receptors in the brain causing encephalitis in some patients. ${ }^{[10]}$

The diagnosis of encephalitis was made based on the personality and behavioral changes associated with altered mental status along with her CSF pleocytosis and high csf protein level. ${ }^{[14]}$ Although it is difficult to prove causality, several clinical features suggest that this syndrome was triggered by ICIs. The timing of the onset of neurologic symptoms after ICI therapy suggests immune-related adverse events rather than classic paraneoplastic neurologic disorders (PNDs). Also, paraneoplastic encephalitis has a subacute or chronic progressive course often preceding the diagnosis of malignancy by several months. The fact that our patient had an irAE involving the liver previously while on ICI may have increased the possibility of autoimmune encephalitis. Our patient had a normal MRI brain; hence the likelihood of paraneoplastic limbic encephalitis is low. Also, she had significant clinical improvement with glucocorticoid therapy and cessation of nivolumab. These observations favor the diagnosis of immune related neurotoxicity. Rechallenge with ICIs after neurologic recovery are considered too risky. ${ }^{[15]}$ 
Treatment of autoimmune encephalitis related to ICIs is based on the severity of adverse effects. For Grade 1 toxicity (mild symptoms) or grade 2 toxicity (new-onset moderate neurologic signs or symptoms), ICIs are withheld and prednisone equivalents at $1 \mathrm{mg}$ to $2 \mathrm{mg} / \mathrm{kg} /$ day are started with a plan for a prolonged steroid taper. Treatment with ICIs is then resumed if the patient improves. In patients with grades 3 to 4 toxicities (immune-mediated encephalitis with confusion and personality changes), ICIs should be discontinued permanently and methylprednisolone $1 \mathrm{gm}$ per day for three to five days should be administered, followed by a glucocorticoid taper. If severe or progressive symptoms are present, IVIG $2 \mathrm{gm}$ per kg per day for five days can be initiated together with methylprednisone. ${ }^{[5,15]}$ Anti-CTLA-4 and anti-PD1 antibodies have a long half-life of 3- 4 weeks, and hence glucocorticoids, if used successfully, should be gradually tapered over a period of several months. The median time to symptoms resolution in steroid responsive patients is 9.4 weeks, although complete recovery may take longer. ${ }^{[6]}$

As elevated levels of tumor necrosis factor alpha and other in- flammatory cytokines are also implicated in the mechanism of neuroinflammation mediated by ICIs, clinicians might consider TNF inhibitors such as infliximab or tocilizumab in steroid non-responsive patients. ${ }^{[5]}$ Alternatively, calcineurin inhibitors such as tacrolimus or cyclosporine can also be used to blunt $\mathrm{T}$ cell responses. ${ }^{[5]}$ Regulatory B cells (Breg) are involved in regulating humoral immunity mediated by follicular helper T cells through PD1/ PD-L1 interactions. Elevated PD-L1 expression on B cells is an important regulator of follicular helper T cell activity. ${ }^{[14]}$ So, in cases of antibody-mediated pathogenesis, patients might respond better to rituximab, intravenous immunoglobulin and plasma exchange. ${ }^{[6,16]}$

Our patient was followed by the oncology department. MRI at 1 month follow up post discharge was normal. Patient was asymptomatic. Plan was made to follow up in another month and to decide further management by the oncology team.

\section{CONFlicts OF INTEREST Disclosure}

The authors have declared no conflicts of interest.

\section{REFERENCES}

[1] Pardoll DM. The blockade of immune checkpoints in cancer immunotherapy. Nature Reviews Cancer. 2012;12(4):252-264. PMid:22437870. https://doi.org/10.1038/nrc3239

[2] Hargadon KM, Johnson CE, Williams CJ. Immune checkpoint blockade therapy for cancer: An overview of FDA-approved immune checkpoint inhibitors. International Immunopharmacology. 2018; 62 , 29-39. PMid:29990692. https://doi.org/10.1016/j.intimp .2018 .06 .001

[3] Postow MA, Sidlow R, Hellmann MD. Immune-related adverse events associated with immune checkpoint blockade: The New England Journal of Medicine. 2018; 378(2): 158-168. PMid:29320654. https://doi .org/10.1056/NEJMra1703481

[4] Naidoo J, Page DB, Li BT, et al. Toxicities of the anti-PD-1 and antiPD-L1 immune checkpoint antibodies. Annals of Oncology. 2015; 26(12): 2375-2391. PMid:26371282 . https ://doi .org/10.109 $3 /$ annonc/mdv383

[5] Blackmon JT, Viator TM, Conry RM. Central nervous system toxicities of anti-cancer immune checkpoint blockade. J Neurol Neuromedicine. 2015; 1(4): 39-45. https ://doi.org/10.29245/2 $572.942 \mathrm{X} / 2016 / 4.1040$

[6] Spain L, Walls G, Julve M, et al. Neurotoxicity from immunecheckpoint inhibition in the treatment of melanoma: a single centre experience and review of the literature. Annals of Oncology. 2016; mdw558. PMid:28426103. https ://doi.org/10.1093/annonc /mdw558

[7] Cuzzubbo S, Javeri F, Tissier M, et al. Neurological adverse events associated with immune checkpoint inhibitors: Review of the literature. European Journal of Cancer. 2015; 73: 1-8. PMid:28064139. https://doi.org/10.1016/j.ejca.2016.12.001

[8] Friedman CF, Proverbs-Singh TA, Postow MA. Treatment of the immune-related adverse effects of immune checkpoint inhibitors: a review. JAMA Oncol. 2016; 2(10): 1346-1353. PMid:27367787.
[9] Vogrig A, Muñiz-Castrillo S, Joubert B, et al. Central nervous system complications associated with immune checkpoint inhibitors. Journal of Neurology, Neurosurgery \& Psychiatry 2020; 91: 772-778. PMid:32312871. https://doi.org/10.1136/jnnp-2020-323 055

[10] Williams TJ, Benavides DR, Patrice KA, et al. Association of autoimmune encephalitis with combined immune checkpoint inhibitor treatment for metastatic cancer. JAMA Neurol. 2016; 73(8): $928 \mathrm{e} 33$. PMid:27271951. https://doi.org/10.1001/jamaneurol. 201 6.1399

[11] Hottinger AF. Neurologic complications of immune checkpoint inhibitors. Curr Opin Neurol. 2016; 29(6): 806-12. PMid:27653290. https://doi.org/10.1097/WC0.0000000000000391

[12] Manousakis G, Koch J, Sommerville RB, et al. Multifocal radiculoneuropathy during ipilimumab treatment of melanoma. Muscle Nerve. 2013; 48(3): 440-444. PMid:23447136. https ://doi .org/ $10.1002 /$ mus . 23830

[13] Thibult M-L, Mamessier E, Gertner-Dardenne J, et al. PD-1 is a novel regulator of human B-cell activation. Int Immunol. 2013; 25(2): 129-137. PMid:23087177. https://doi.org/10.1093/intimm /dxs098

[14] Guan H, Wan Y, Lan J, et al. PD-L1 is a critical mediator of regulatory B cells and T cells in invasive breast cancer. Sci Rep. 2016. PMid:27762298. https ://doi.org/10.1038/srep35651

[15] Brahmer JR, Lacchetti C, Schneider BJ, et al. Management of immune-related adverse events in patients treated with immune checkpoint inhibitor therapy: American Society of Clinical Oncology Clinical Practice Guideline. J Clin Oncol. 2018; 36: 1714-1768. PMid:29442540. https ://doi .org/10.1200/JC0.2017.77.63 85

[16] Opdivo [package insert]. Princeton, NJ: Bristol-Myers Squibb Company; 2016. 